\title{
Noise Reduction in Leaf Image by Fuzzy Based Filtering Technique
}

\author{
Ajitha $\mathbf{N}^{1}$, Nandhini $\mathbf{S}^{2}$ \\ Assistant Professor, Computer Science, Sri Krishna Arts and Science College, Coimbatore, India ${ }^{1}$ \\ Assistant Professor, Computer Technology, Sri Krishna Arts and Science College, Coimbatore, India ${ }^{2}$
}

\begin{abstract}
The accuracy in recognition and classification of the plant disease is highly significant for the successful farming of crop and this can be performed using image processing. This research is conversed various methods to segment the disease portion of the plant. This study also discussed some noise reduction techniques and image segmentation techniques for identification of infected leaf and the categorization of plant diseases. Image segmentation is the method of partitioning a digital image into numerous segments. The outcome of image segmentation is a group of segments that jointly cover the whole image, or a collection of contours acquired from the image. With regard to features namely texture, color and intensity every pixel in a region is similar.
\end{abstract}

Keywords: Segmentation, Fuzzy, Speckle, Histogram, Noise, Impulse, Thresholding

\section{INTRODUCTION}

Noise is an unplanned variation of picture Intensity and visible as an element of grains in the picture. It may form to arise in the picture as effects of fundamental physics like photon environment of light or thermal value of heat privileged in the image sensors (Rohit \& Jahid 2013). It may create at the time of taking or image communication. Noise denotes, the pixels in the picture show varied intensity values alternate to true pixel entry that are received from image. Noise elimination algorithm is the method of removing or decreasing the noise from the picture. The noise removal techniques reduce or eliminate the visibility of noise by smoothing the whole image leaving areas nearby contrast boundaries. But these techniques can vague fine, low contrast details (EmreCelebi et al 2007). The usual types of noise that rises in the image are: a) Impulse noise, b) Preservative noise (Parminder \& Jagroop 2011) c) Multiplicative noise. Varied noises have their own features which make them different from others. Image noise can likewise devise in film grain and in the inevitable shot noise of a perfect photon detector. Image noise is an unwanted by-product of image acquired.

\section{DIFFERENT SOURCES OF NOISE}

Noise is included in the image at the period of image attainment or transmission. Various factors may be accountable for introduction of noise in the picture. The number of pixels tainted in the image will elect the quantification of the noise. The primary causes of noise in the digital picture are: a) The imaging sensor may be effected by environmental conditions while image acquisition. b)Inadequate Light levels and sensor temperature may inculcate the noise in the image. c) Intrusion in the transmission passage may also spoil the image. d) If dust particles exist on the scanner screen, it may also introduce noise in the picture.

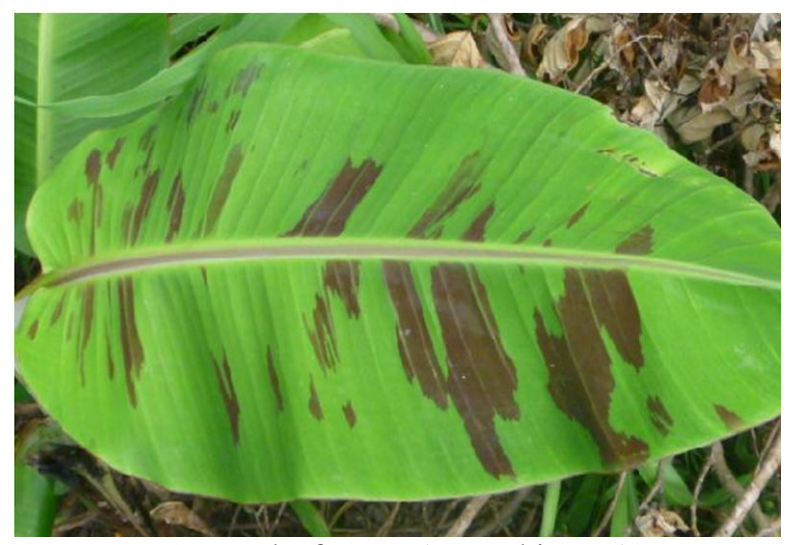

Banana leaf Image(normal image) 


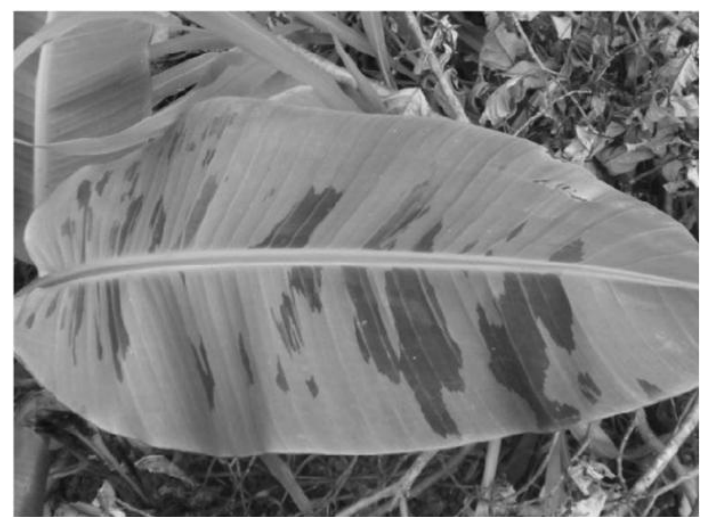

Banana leaf Image(grey scale image)

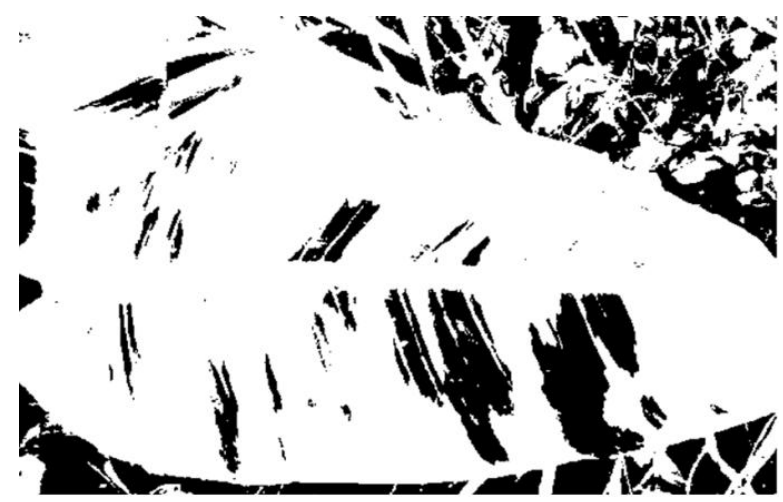

Banana leaf Image (thresholded image)

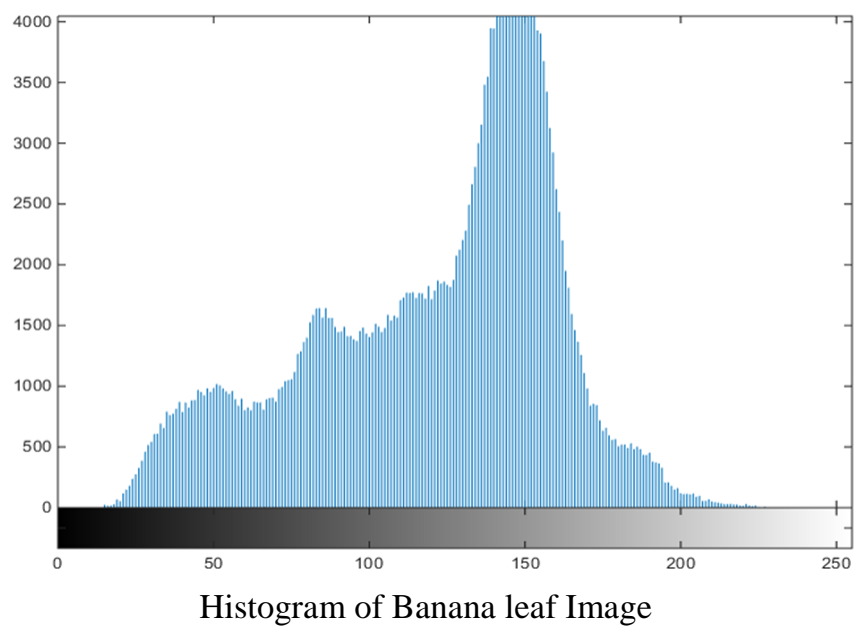

III. THRESHOLDING

Thresholding methods are classified into six different types depending on the information-manipulation technique.

1. Histogram shape-based technique: This method analyzes curvatures, peaks, valleys of the smoothed histogram.

2. Clustering-based method: Gray-level samples are clustered in two sections as background and foreground entities, or alternately are structured as a mixture of two Gaussians in this technique.

3. Entropy-based method: This method is based on the entropy of the foreground and background area, the crossentropy between the actual and digitized image etc.

4. Object Attribute-based method: This method finds a measure of similarity between the gray-state and the digitized images, namely fuzzy shape similarity, edge coincidence etc.

5. Spatial method: A higher-order probability distribution is applied with correlation between neighboring pixels.

6. Local method: Threshold value with local method maps the every pixel to the local image features. In this method, a different $\mathrm{T}$ (Threshold) is chosen for each pixel in the image. 


\section{International Journal of Advanced Research in Computer and Communication Engineering}

Vol. 7, Issue 11, November 2018

\section{TYPES OF NOISE}

Noise is said to be a degradation in the picture signal created by external disturbance .If an image is being directed electronically from one location to another through satellite or wireless communication or through networked chains, it is expected to have errors to happen in the image signal. These faults will appear on the picture output in various ways depending on the category of disturbance in the signal. Normally it is known like what type of errors to assume and the category of noise on the picture; hence it is required to examine some of the standard noise for removing or decreasing noise in color image. Image Noise is categorized as Quantization noise (uniform noise), Speckle noise (Multiplicative noise), Amplifier noise (Gaussian noise), Salt-and-pepper noise (Impulse noise), Film grain, on-isotropic noise, Shot noise and Periodic noise.

1. Amplifier Noise (Gaussian noise): The standard method of amplifier noise is addition, Gaussian, reliant on at each pixel and reliant on of the signal intensity, sourced primarily by Johnson-Nyquist noise (thermal noise), involving that which initiates from the reset noise of capacitors ("kTC noise"). This is a perfect form of white noise, which is created by random differences in the signal. In color cameras where high amplification is applied in the blue color passage than in the green or red path, there can be higher noise in the blue passage. Amplifier noise is a main part of the noise of a picture sensor, which is, of the continuous noise level in dark regions of the image. In Gaussian noise, every pixel in the image will be converted from its original value through a (usually) small amount(Vijay et al 2007). A histogram, a design of the quantity of distortion of a pixel point against the frequency with which it happens, shows a usual distribution of noise. Whereas other distributions are probable, the Gaussian (normal) distribution is normally a good model, because of the central limit theorem that states that the sum of varied noises tends to attain a Gaussian distribution. Not only this but also Gaussian noise signifies statistical noise taking probability density function (PDF) equivalent to the normal distribution, which is also named as the Gaussian distribution. In other terms, the values that the noise can include on are Gaussian distributed. A special situation is white Gaussian noise, shows that the values at any duo of times are equally distributed and statistically autonomous in communication path testing and modelling, Gaussian noise is applied as additive white noise to produce additive white Gaussian noise (Stefan Schulte et al 2007). In signal handling, white noise is an arbitrary signal with a fixed power spectral density. The term is applied, with this or similar values, in many scientific and technical areas, involving physics, acoustic engineering.

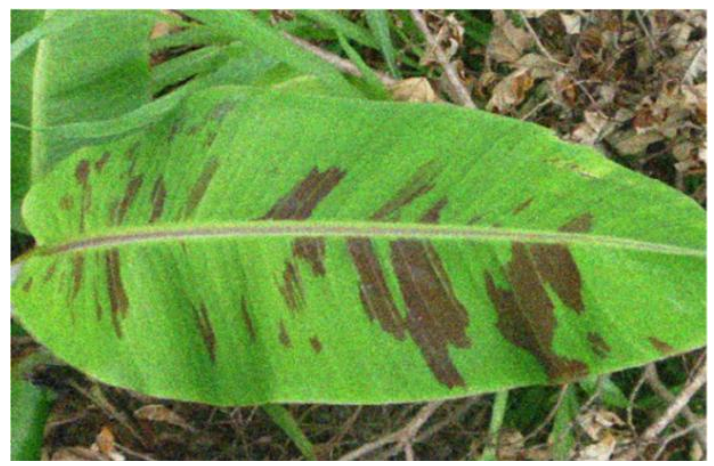

Gaussian Noise Image

2. Salt-and-Pepper Noise (Impulse Noise): Salt and pepper noise is occasionally called impulse noise or spike noise or arbitrary noise or independent noise. In salt and pepper noise picture elements in the image are highly different in color or intensity disparate their surrounding picture element. Salt and pepper deprivation can be created by sharp and sudden disturbance in the picture signal.

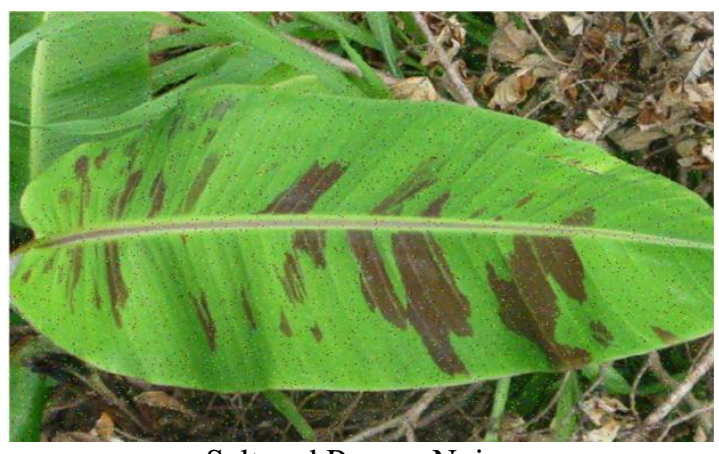

Salt and Pepper Noise 


\section{International Journal of Advanced Research in Computer and Communication Engineering}

Vol. 7, Issue 11, November 2018

Usually this type of noise will only disturb a small range of image pixels. When observed, the image contains dark and white dots, henceforth the term salt and pepper noise. Typical causes include flecks of dust located inside the camera and overheated or defective (Charge-coupled device) CCD elements. A picture containing salt-and-pepper noise will contain dark pixels in bright areas and vice versa. This category of noise can be produced by dead pixels that are named as impulsive noise. It presences is randomly scattered white or black pixel above the image. It occasionally happens for memory cell failure, for synchronization faults in image digitizing or communication. This category of noise can be created by analog to digital converter errors and bit error during transmission.

3. Shot Noise: The leading noise in the lighter portions of an image from an image sensor is classically that created by statistical quantum fluctuations, that is, variation in the count of photons sensed at a specified exposure level; this noise is named as photon shot noise. Shot noise consists of a root mean-square value proportional to the square root of the picture intensity, and the noises at different pixels are liberated of one another. Shot noise trails a Poisson distribution, which is normally not very variance from Gaussian. Enhancing of photon shot noise, there can be extra shot noise from the dark leakage current in the picture sensor; this noise is also known as "dark shot noise" or "dark-current shot noise".

4. Quantization Noise (Uniform Noise): The noise created by quantizing the picture element of a sensed image to a count of discrete levels is named as quantization noise; it has a roughly uniform distribution, and can be signal dependent, though it will be signal independent if additional noise sources are huge that cause dithering, or if dithering is explicitly used.

5. Film Grain: In association with shot noise, grain of photographic film is a signal-dependent noise. That is, if film grains were evenly distributed (equal number per area), and if every grain has an equivalent and independent probability of creating a dark silver grain afterward absorbing photons, then the count of such dark grains in a location will be random with a binomial distribution; in locations where the probability is less, this distribution will be nearer to the classic Poisson distribution of shot noise; nonetheless a simple Gaussian distribution is often applied as an accurate model.

6. Speckle Noise (Multiplicative Noise): Speckle is a coarse 'noise' that inherently occurs in and degrades the value of the dynamic radar and synthetic aperture radar (SAR) images (Aborisade 2011). Speckle noise in conservative radar consequences from random fluctuations in the return signal from an entity that is no larger than a distinct imageprocessing element. It upsurges the mean grey level of a native area. Speckle noise in SAR is usually more serious, creating difficulties for image interpretation. It is created by coherent functioning of backscattered signals from several distributed targets. In SAR oceanography, for instance, speckle noise is created by signals from basic scatters, the gravity-capillary ripples, and establishes as a pedestal image, underneath the picturee of the sea waves. Synthetic Apertures Radar (SAR) technique is prevalent because of its applicability under various weather conditions, its capability to pierce through clouds and soil. A SAR image is a mean intensity approximation of the radar reflectivity of the location which is being imaged. Speckle noise in such scheme is to be referred as the variance between a measurement and the exact mean value. Degraded image with speckle noise in ultrasound imaging is presented by the equation.

$$
\mathrm{G}(\mathrm{n}, \mathrm{m})=\mathrm{f}(\mathrm{n}, \mathrm{m}) * \mathrm{u}(\mathrm{n}, \mathrm{m})+\xi(\mathrm{n}, \mathrm{m})
$$

Where $g(n, m)$ is the observed image. $u(n, m)$ is the multiplicative element and $\xi(n, m)$ is the $\xi(n, m)$ is the extra component of the speckle noise. While Gaussian noise can be demonstrated by random values included to an image, speckle noise can be structured by random values multiplied by pixel values henceforth it is also named multiplicative noise. Speckle noise is a main problem in certain radar applications.

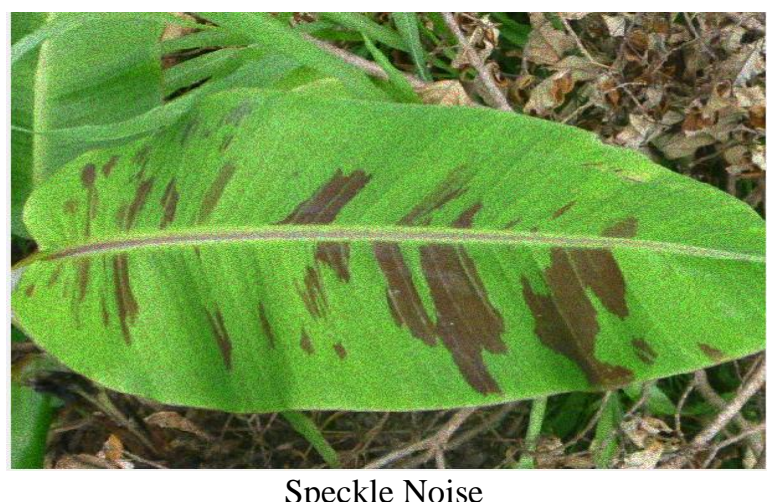

Speckle Noise 
Vol. 7, Issue 11, November 2018

\section{REMOVING NOISE FROM IMAGES BY FILTERING}

Image noise is an obvious side-effect happening as a result of image acquisition, more simply defined as inaudible, yet expected fluctuations. In a digital camera, if the light that pierces the lens misaligns with the sensors, it might develop image noise. Even though noise is not so clearly visible in a picture, a similar kind of image noise is bound to subsist. Each type of electronic device acquires and transmits little noise and sends it on to what it is developing. While the images are broadcasted over channels, they are contaminated with impulse noise because of noisy channels. This impulse noise contains of large positive and negative spikes. The positive spikes possess values much higher than the background and thus it is shown as bright spots, whereas the negative spikes contain values less than the background and it is shown as darker spots. Both the portions for the positive and negative spikes are evident to the human eye. In addition, Gaussian style of noise influences the image. Thus, filters are necessary for removing noises prior to processing. There are several types of filters in the paper to eliminate noise. They are of several kinds namely wiener filter, linear smoothing filter, median filter and Fuzzy filter. In these filtering methods, the three primaries (Red, Green and Blue) are done individually. It is trailed by some gain to recompense for attenuation obtained from the filter. The filtered outcomes are then joined to form the coloured image. It involves a simple process.

1. Linear Filters: Linear filter is applied to remove certain category of noise. Averaging or Gaussian filters are suitable for this function. Linear filters are inclined to blur sharp boundaries, obliterate lines and other fine image particulars, and achieve poorly in the existence of signal-dependent noise. Example, the Gaussian mask includes elements described by a Gaussian function. This convolution fetches the value of every pixel into nearer harmony with the values of its associates. Generally, a smoothing filter assigns each pixel to the mean value, or a weighted mean, of itself and its closer neighbors; the Gaussian filter is presently one possible group of weights. Smoothing filters is inclined to blur an image, since pixel intensity values that are considerably higher or less than the surrounding neighborhood might "smear" crosswise the region. Since this blurring, linear filters are seldom applied practically for noise reduction; they are, still, often applied as the foundation for nonlinear noise reduction filters.

2. Adaptive Filter: The wiener function uses a Wiener filter (a type of linear filter) to an picture adaptively, preparing itself to the local image variance. If the difference is large, wiener achieves little smoothing. If it is less, wiener performs high smoothing. This method often produces better outcomes than linear filtering. The adaptive filter is highly selective than a analogous linear filter, conserving edges and other high-frequency portions of an image. Additionally, there are no design activities; the wiener 2 function performs all preliminary computations and calculates the filter for a given picture. Wiener2, though, does need more computation time than linear filtering. Wiener works efficiently when the noise is constant-power ("white") additive noise, such as Gaussian noise. An additional method for eradicating noise is to evolve the image beneath a smoothing partial differential equation alike the heat equation which is named as anisotropic diffusion.

3. Non -linear filter: In current years, a variety of nonlinear median category filters namely weighted median, rank accustomed rank selection, and undisturbed median have been created to overcome this is inadequacy. To execute a median filter: 1.Consider every pixel in the image. Sort the neighboring pixels into order dependent upon their strengths. To replace the unique value of the picture elements with the median value from the list, a median filter is a rank selection (RS) filter, a predominantly harsh member of the group of rank-conditioned rank-selection (RCRS) filters; a much milder associate of that family, is sometimes chosen, especially in photographic functions. Median and other RCRS filters are best at eliminating salt and pepper noise from an image, and also create relatively little blurring of ends, and hence are frequently used in computer vision functions. Median filtering is analogous to using an averaging filter, in that each output pixel is assigned to an average of the pixel values in the neighborhood of the corresponding input pixel. Though, with median filtering, the value of an output pixel is computed by the median of the neighborhood pixels, rather than the mean.

\section{ALGORITHM OF MEDIAN FILTER}

The algorithm for the median filter is as given below:

Step 1. Choose a two dimensional window Wind of size $4 * 4$.

Presume that the pixel being executed is $\mathrm{Pa}, \mathrm{b}$.

Step 2. Calculate Windmed the median of the pixel values in window Wind.

Step 3. Replace Pa,b by Windmed.

Step 4. Repeat steps 1 to 3 till all the pixels in the whole image are executed. 


\section{International Journal of Advanced Research in Computer and Communication Engineering}

Vol. 7, Issue 11, November 2018

\section{Advantage}

i) Implement is simple.

ii) Applied for de-noising different categories of noises.

\section{Disadvantage}

i) Median Filter is inclined to remove image details whilst decreasing noise such as thin lines and corners.

ii) Performance of median filtering is not adequate in case of signal dependent noise. To eliminate these difficulties diverse variations of median filters have been designed for the better results.

\section{PROPOSED FUZZY BASED FILTERING (FBF)}

The proposed method involves fuzzy based median filter that reduces noise efficiently.

\section{EXPERIMENTAL STUDIES}

To validate the proposed FBF, simulation is performed on leaf images. The image is subjected with $10 \%$ as low density noise to a highest of $95 \%$ noise density. The proposed method is compared with existing methods using MATLAB R2008a.

Input: Noise image NI

Output: Filtered image FI

\section{Algorithm}

Step 1: Initialize a sub-window size, $\mathrm{Windz}=4$ and maximum window size, Windmax $=10$.

Step 2: Chose a sub-window WindzxWindz with fuzzy measure Ma,b.

$\mathrm{Ma}, \mathrm{b}=\mathrm{P}_{\mathrm{a}+\mathrm{m}, \mathrm{b}+\mathrm{n}} \mid \mathrm{m}, \mathrm{n} \in\left((\mathrm{Ma}, \mathrm{b})-\frac{\mathrm{T}}{\max -\min }\right.$

Step 3: Compute the maximum (Mmax) and minimum (Mmin) grey values within the window

Step 4: If the $|\operatorname{Mmax}-\mathrm{Mmin}| \leq$ Threshold (T), then go to step 9.

Step 5: If the Mmin < Mi,j. < Mmax then go to step 9.

Step 6: Collect the set of pixels (P) from the sub-window where, Mmin $<\mathrm{Pa}, \mathrm{b} .<\mathrm{Mmax}$

Step 7: If the size of $P \geq$ Wind, then replace with fuzzy median Ma,b of pixels in $P$.

Go to step 9.

Step 8: Windz $\square \square$ Windz +2 ,

If Wind $\leq$ Windmax ,then go to Step 2.

else

replace the center pixel by mean of the pixels in sub-window of size Windmax

Step 9: Shift the test pixel. Go to Step 1

Step 10: Repeat Steps 1 through 9 for image pixels.

Comparative assessment of PSNR(dB) for various filters on leaf image

\begin{tabular}{|c|c|c|c|}
\hline \% of Noise & Standard Median Filter(SMF) & Adaptive Median Filter(AMF) & Fuzzy Based Filter(FBF) \\
\hline 10 & 29.7438 & 33.3454 & 37.8264 \\
\hline 20 & 27.6729 & 38.4930 & 42.8862 \\
\hline 30 & 23.8284 & 29.3492 & 31.3725 \\
\hline 40 & 19.3240 & 25.5426 & 27.6352 \\
\hline 50 & 15.8302 & 19.5673 & 20.5274 \\
\hline 60 & 12.9384 & 17.4956 & 19.2735 \\
\hline 70 & 9.9937 & 15.4782 & 20.5927 \\
\hline 80 & 7.3847 & 11.3836 & 15.2888 \\
\hline 90 & 6.5848 & 10.4934 & 18.8372 \\
\hline
\end{tabular}

Table shows that the proposed fuzzy based filtering technique presents considerably better results when compared with standard median filter and adaptive median filter technique 
Vol. 7, Issue 11, November 2018

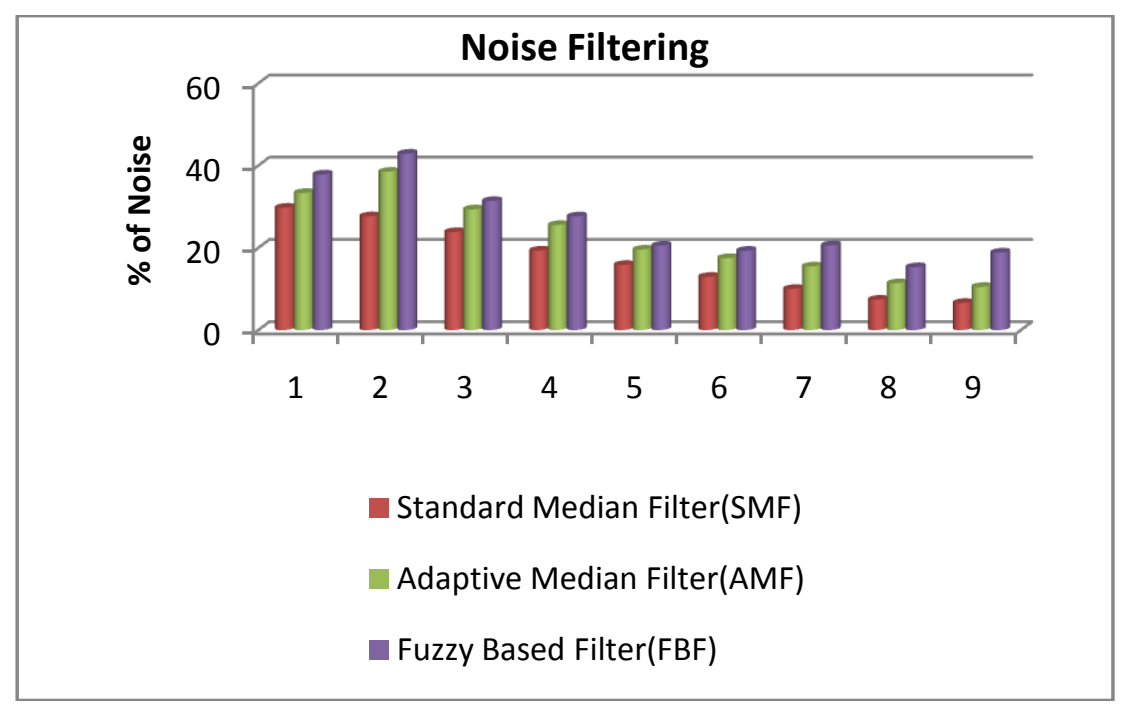

\section{CONCLUSION}

In this paper, various filtering techniques were discussed for removing noises in color images. Furthermore, the proposed filtering technique is presented and compared results for these filtering techniques. The results obtained applying fuzzy based filtering technique guarantees noise free and quality of the image as well. The primary advantage of this fuzzy based filter is the fuzzy measure that acquires the color component differences.

\section{REFERENCES}

[1]. Rohit Verma and Dr.Jahid Ali .A comparative study of various types of image noise and efficient noise removal techniques .International Journal of Advanced Research in Computer Science and Software Engineering,Volume 3,October2013.

[2]. Parminder Kaur and Jagroop Singh. 2011.A Study Effect of Gaussian Noise on PSNR Value for Digital Images International journal of computer and electrical engineering Vol. 3, No. 2,1793-8163.

[3]. V.R.Vijay Kumar, S.Manikandan, D.Ebenezer, P.T.Vanathi and P.Kanagasabapathy.2007.High Density Impulse noise Removal in Color Images Using Median Controlled Adaptive Recursive Weighted Median Filter. IAENG International Journal of computer.

[4]. Stefan Schulte, Valérie De Witte, and Etienne E. Kerre.2007. A Fuzzy Noise Reduction Method for IEEE Transactions On Image Processing, Vol.16.No511436.

[5]. Aborisade, D.O. 2011 A Novel Fuzzy logic Based Impulse Noise Filtering Technique, International Journal of Advanced Science and Technology Vol.32.

[6]. Orlando.J,Tobias.2004. Image segmentation by histogram thresholding using fuzzy sets. IEE transaction on image processing, Vol.11, No.12. 